\title{
Stability of Equilibria for Piecewise-linear Models of Genetic Regulatory Networks
}

\author{
Richard Casey Hidde de Jong Jean-Luc Gouzé
}

\begin{abstract}
A formalism based on piecewise-linear (PL) differential equations has been shown to be well-suited to modelling genetic regulatory networks. The discontinuous vector field inherent in the PL models leads to the approach of Filippov, which extends the vector field to a differential inclusion. We study the stability of equilibria (called singular equilibrium sets) that lie on the surfaces of discontinuity. We prove several theorems that characterize the stability of these singular equilibria directly from the state transition graph, which is a qualitative representation of the dynamics of the system.
\end{abstract}

\section{INTRODUCTION}

A class of piecewise-linear (PL) models, originally proposed by Glass and Kauffman [11], has been widely used in modelling genetic regulatory networks. The properties of these PL models have been well-studied in the mathematical biology literature, by for example Glass and Pasternack [12], Plahte et al [16], Thomas et al [19], Edwards [8], Gouzé and Sari [13], and more recently in the hybrid systems literature by Ghosh and Tomlin [10], Alur and Belta [1], and Belta et al [2]. The variables in the piecewise-linear differential equation (PLDE) models are the concentrations of proteins encoded by the genes, while the differential equations describe the regulatory interactions in the network by means of step functions. The use of step functions is motivated by the switch-like behaviour of many of the interactions in genetic regulatory networks [17], but it does lead to some difficulties. The vector field for the PLDE model is undefined when one of the variables assumes a value where the step function is discontinuous, referred to as a threshold value. Recent work by Gouzé and Sari [13] uses an approach due to Filippov [9] to define the solutions on the threshold hyperplanes. The approach involves extending the PLDE to a piecewise-linear differential inclusion (PLDI).

The study of the stability of equilibria in switching domains (singular equilibria) for PL models of genetic regulatory networks is the focus of this paper. The stability of equilibria for PL systems is an important subject in hybrid systems and control theory. See, for example, the review of Decarlo et al [6]. We here give several results on the stability of singular equilibria based on properties of the state transition graph.

Due to space limitation, some of the proofs are not included and can be found in [3].

R. Casey and J.-L. Gouzé are with COMORE INRIA, Unité de recherche Sophia Antipolis, 2004 route des Lucioles, BP 93, 06902 Sophia Antipolis, France, gouze@sophia.inria.fr

H. de Jong is with HELIX INRIA, Unité de recherche Rhône-Alpes, 655 avenue de l'Europe, Montbonnot, 38334 Saint Ismier Cedex, France.

\section{PieCEWise-LineAr Models of Genetic REGULATORY NETWORKS}

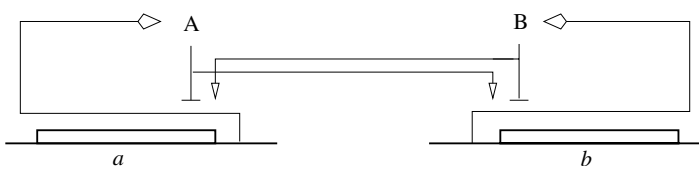

Fig. 1. Example of a genetic regulatory network of two genes ( $a$ and $b$ ), each coding for a regulatory protein (A and $\mathrm{B})$. The notation follows in a simplified form the conventions of Kohn [14].

The schematic diagram in Figure 1 describes a simple genetic regulatory network. In this example, the genes $a$ and $b$ code for the proteins $\mathrm{A}$ and $\mathrm{B}$, which in turn control the expression of the two genes $a$ and $b$. Protein A inhibits gene $a$ and activates gene $b$ above certain threshold concentrations, which are assumed to be different. Similarly protein B inhibits gene $b$ and activates gene $a$ above different threshold concentrations.

The dynamics of genetic regulatory networks can be modelled by a class of dynamical systems proposed originally by Glass and Kauffman [11]. The model has the general form

$$
\dot{x}_{i}=f_{i}(x)-\gamma_{i} x_{i}, \quad 1 \leq i \leq n,
$$

where $x=\left(x_{1}, \ldots, x_{n}\right)^{t}$ is a non-negative vector of protein concentrations. The non-negative quantities $f_{i}(x)$ and $\gamma_{i} x_{i}$ represent synthesis and degradation rates for each protein $x_{i}$ respectively. We can write the system (1) more compactly as

$$
\dot{x}=f(x)-\gamma x,
$$

where $f(x)=\left(f_{1}(x), \ldots, f_{n}(x)\right)^{t}$ and $\gamma=\operatorname{diag}\left(\gamma_{1}, \ldots, \gamma_{n}\right)$ is a constant diagonal matrix.

The functions $f_{i}: \mathbb{R}_{+}^{n} \rightarrow \mathbb{R}_{+}$represent the dependence of the rate of synthesis of a protein encoded by gene $i$ on the concentrations $x$ of protein in the cell. They are defined by a sum of terms $\kappa_{i l} b_{i l}(x)$, where $\kappa_{i l}>0$ is a rate parameter, and $b_{i l}: \mathbb{R}_{+}^{n} \rightarrow\{0,1\}$ is a boolean-valued regulation function. The regulation functions $b_{i l}$ capture the conditions under which the protein encoded by gene $i$ is synthesized at a rate $\kappa_{i l}$. These conditions are written down as combinations of step functions $s^{+}, s^{-}$where

$s^{+}\left(x_{i}, \theta_{i}\right)=\left\{\begin{array}{ll}1, & x_{i}>\theta_{i}, \\ 0, & x_{i}<\theta_{i},\end{array}\right.$ and $s^{-}\left(x_{i}, \theta_{i}\right)=1-s^{+}\left(x_{i}, \theta_{i}\right)$

Here $x_{i}$ is a component of the concentration vector $x$ for the proteins, and the parameters $\theta_{i}$ are threshold concentrations. The use of such step functions has been 
motivated by the observation that the activity of a gene changes in a switch-like manner at a threshold concentration of a regulatory protein. The model (2) is piecewise-linear (PL) with the above definition of the $f_{i}$. Note that the step functions are not defined for $x_{i}=\theta_{i}$, so neither are the regulation functions.

The equations modelling the example network in Figure 1 can be written down as

$$
\begin{aligned}
& \dot{x}_{a}=\kappa_{a} s^{+}\left(x_{b}, \theta_{b}^{1}\right) s^{-}\left(x_{a}, \theta_{a}^{2}\right)-\gamma_{a} x_{a}, \\
& \dot{x}_{b}=\kappa_{b} s^{+}\left(x_{a}, \theta_{a}^{1}\right) s^{-}\left(x_{b}, \theta_{b}^{2}\right)-\gamma_{b} x_{b} .
\end{aligned}
$$

Gene $a$ is expressed at a rate $\kappa_{a}$ if the concentration $x_{b}$ of protein $\mathrm{B}$ is above the threshold $\theta_{b}^{1}$ and the concentration $x_{a}$ of protein $\mathrm{A}$ is below the threshold $\theta_{a}^{2}$. Similarly, gene $b$ is expressed at a rate $\kappa_{b}$ if the concentration $x_{a}$ of protein A is above the threshold $\theta_{a}^{1}$ and the concentration $x_{b}$ of the protein $\mathrm{B}$ is below the threshold $\theta_{b}^{2}$.

\section{A. Domains in Phase Space}

The dynamics of the piecewise-linear system (2) can be studied in the $n$-dimensional phase space $\Omega=\Omega_{1} \times \ldots \times \Omega_{n}$, where each $\Omega_{i}$ is defined by $\Omega_{i}=\left\{x_{i} \in \mathbb{R}_{+} \mid 0 \leq x_{i} \leq\right.$ $\left.\max _{i}\right\}$, for some positive parameter $\max _{i}$, with $\max _{i}>$ $\max _{x \in \Omega}\left(f_{i}(x) / \gamma_{i}\right)$. A protein encoded by a gene will be involved in different interactions at different concentration thresholds, so for each variable $x_{i}$ we assume there are $p_{i}$ ordered thresholds $\theta_{i}^{1}, \ldots, \theta_{i}^{p_{i}}$. The $(n-1)$-dimensional hyperplanes defined by these thresholds partition $\Omega$ into hyper-rectangular regions we call domains. Specifically, a domain $D \subset \Omega$ is defined to be a set $D=D_{1} \times \cdots \times D_{n}$, where each set $D_{i}$, for $1 \leq i \leq n$, is given by one of the following equations

$$
\begin{aligned}
D_{i} & =\left\{x_{i} \in \Omega_{i} \mid 0 \leq x_{i}<\theta_{i}^{1}\right\}, \\
D_{i} & =\left\{x_{i} \in \Omega_{i} \mid x_{i}=\theta_{i}^{1}\right\}, \\
D_{i} & =\left\{x_{i} \in \Omega_{i} \mid \theta_{i}^{1}<x_{i}<\theta_{i}^{2}\right\}, \\
D_{i} & =\left\{x_{i} \in \Omega_{i} \mid x_{i}=\theta_{i}^{2}\right\}, \\
& \vdots \\
D_{i} & =\left\{x_{i} \in \Omega_{i} \mid x_{i}=\theta_{i}^{p_{i}}\right\}, \\
D_{i} & =\left\{x_{i} \in \Omega_{i} \mid \theta_{i}^{p_{i}}<x_{i} \leq \max _{i}\right\} .
\end{aligned}
$$

Let $\mathcal{D}$ denote the set of all domains in $\Omega$. A domain $D \in \mathcal{D}$ is called a regulatory domain if none of the variables $x_{i}$ has a threshold value in $D$. In contrast, a domain $D \in \mathcal{D}$ is called a switching domain if at least one of the variables has a threshold value in $D$. The corresponding variables $x_{i}$ are called switching variables in $D$. For convenience, we denote the sets of regulatory and switching domains by $\mathcal{D}_{r}$ and $\mathcal{D}_{s}$ respectively. Following Mestl et al [15], we define the order of a switching domain to be the number of switching variables in that domain.

Definition 1: The order of a domain $D \in \mathcal{D}$ is the number $k \in \mathbb{N}, 0 \leq k \leq n$ equal to the number of switching variables in $D$, denoted order $(D)$.
Definition 2: For every domain $D \in \mathcal{D}_{s}$ of order $k \geq 1$, define $\operatorname{supp}(D) \subset \Omega$ to be the $(n-k)$-dimensional hyperplane containing $D$. If $D \in D_{r}$ then we define $\operatorname{supp}(D)$ to be equal to $\Omega$.

For every domain $D$ we also define the boundary $\partial D$ of $D$ in $\operatorname{supp}(D)$ and the sets

$$
\begin{aligned}
& A(D)=\left\{D^{\prime} \in \mathcal{D} \mid D^{\prime} \subseteq \partial D\right\}, \text { and } \\
& R(D)=\left\{D^{\prime} \in \mathcal{D}_{r} \mid D \subseteq \partial D^{\prime}\right\} .
\end{aligned}
$$

Two domains $D$ and $D^{\prime}$ will be said contiguous if $D \in$ $A\left(D^{\prime}\right)$ or $D^{\prime} \in A(D)$.

\section{B. Classical Solutions and Focal Points}

For any regulatory domain $D \in \mathcal{D}_{r}$, the function $f(x)$ is constant for all $x \in D$, and it follows that the piecewiselinear system (2) can be written as a linear vector field

$$
\dot{x}=f^{D}-\gamma x, \quad x \in D,
$$

where $f^{D}$ is constant in $D$. Restricted to $D$, this is a classical linear ordinary differential equation. Clearly $x(t) \rightarrow \phi(D)$ monotonically as $t \rightarrow \infty$, until $x(t)$ reaches the boundary of the regulatory domain $D$.

Definition 3: Given a regulatory domain $D \in \mathcal{D}_{r}$, the point $\phi(D)=\gamma^{-1} f^{D} \in \Omega$ is called the focal point for the flow in $D$.

Generally we make the assumption that $\phi(D) \notin \operatorname{supp}\left(D^{\prime}\right)$, for all $D^{\prime} \subseteq \partial D$, for otherwise solutions can take infinite time to reach a focal point in the boundary of their domain. This is a special case of a more general assumption we make in Section 2.3. What happens when a solution leaves a regulatory domain $D$ and enters a switching domain in the boundary of $D$ ? Since the step functions are not defined when a variable $x_{i}$ takes some threshold value $\theta_{i}^{q_{i}}$, the vector field is undefined on the switching domains.

\section{Filippov Solutions and Focal Sets}
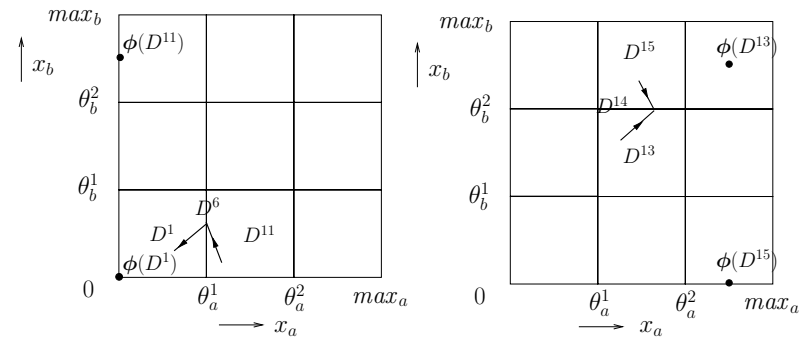

Fig. 2. Behavior of the system of Figure 1 at some of the thresholds The domains $D^{1}, D^{6}, D^{11}, D^{13}, D^{14}$ and $D^{15}$ are shown, in addition to the focal points $\phi\left(D^{1}\right)=(0,0), \phi\left(D^{11}\right)=\left(0, \kappa_{b} / \gamma_{b}\right), \phi\left(D^{13}\right)=$ $\left(\kappa_{a} / \gamma_{a}, \kappa_{b} / \gamma_{b}\right)$ and $\phi\left(D^{15}\right)=\left(\kappa_{a} / \gamma_{a}, 0\right)$. (a) The solution trajectories in $D^{11}$ can be continued in $D^{1}$, so $D^{6}$ is a transparent wall, whereas in (b), the solution trajectories in $D^{13}$ cannot be continued in $D^{15}$. The behavior of the system in $D^{14}$ cannot be determined without a Filippov-like approach.

In order to define the solutions on switching domains, we use a construction originally proposed by Filippov [9] and recently applied to PL systems of this form [13], [5]. The Filippov approach is classical in control theory, where the 
solutions on switching domains are known as sliding modes [7], [20]. The method consists of extending the system (6) to a differential inclusion,

$$
\dot{x} \in H(x)
$$

where $H$ is a set-valued function. If $D$ is a switching domain, for $x \in D$, then

$$
H(x)=\overline{c o}\left(\left\{f^{D^{\prime}}-\gamma x \mid D^{\prime} \in R(D)\right\}\right),
$$

where we recall $R(D)=\left\{D^{\prime} \in \mathcal{D}_{r} \mid D \subseteq \partial D^{\prime}\right\}$ is the set of all regulatory domains with $\mathrm{D}$ in their boundary, and $\overline{c o}(X)$ is the closed convex hull of $X$. For switching domains, $H(x)$ is generally multi-valued so we define solutions of the differential inclusion as follows.

Definition 4: An absolutely continuous function $\xi_{t}\left(x_{0}\right)$ is a solution of (7) on $[0, T]$ in the sense of Filippov if $\xi_{0}\left(x_{0}\right)=$ $x_{0}$ and $\dot{\xi}_{t} \in H\left(\xi_{t}\right)$, for almost all $t \in[0, T]$.

Hereafter we will usually refer to "solutions in the sense of Filippov" as "Filippov solutions" or simply as "solutions" when discussing solutions of the differential inclusion $\dot{x} \in$ $H(x)$.

It is useful to define a concept analogous to the focal points defined for regulatory domains, extended to deal with switching domains.

Definition 5: Let $D \in \mathcal{D}$ be a domain. If $D$ is a regulatory domain then its focal set $\Phi(D)$ is given by $\Phi(D)=\{\phi(D)\}$, where $\phi(D)$ is the focal point of $D \in \mathcal{D}_{r}$ as in Definition 3 . If $D$ is a switching domain of order $k$, and $\operatorname{supp}(D)$ is the $(n-k)$-dimensional hyperplane supporting $D$, then its focal set $\Phi(D)$ is

$$
\Phi(D)=\operatorname{supp}(D) \cap \overline{c o}\left(\left\{\phi\left(D^{\prime}\right) \mid D^{\prime} \in R(D)\right\}\right) .
$$

Thus $\Phi(D)$ can be a singleton, but more generally is a closed convex bounded set and hence is referred to as a focal set. The solutions that remain in a switching domain are called sliding modes. These sliding modes can occur in $D$ when $\Phi(D)$ is non-empty [13].

Further, for $D \in \mathcal{D}_{s}$, if $\Phi(D) \neq\{\}$ and $\Phi(D) \cap D=\{\}$, then all solutions will eventually leave the switching domain. If $\Phi(D) \neq\{\}$ and $\Phi(D) \cap D \neq\{\}$, then there are points in $D$ that behave like a set of equilibrium points in the classical sense. Under what conditions a set $\Phi(D)$ with $\Phi(D) \cap D \neq$ \{\} will be stable in some sense is the subject of the remainder of the paper.

For a switching domain $D$ with $\Phi(D) \neq\{\}$, all solutions converge towards $\Phi(D)$ in a restricted sense. The convergence is monotonic in the sense that for all components $\left(\xi_{t}\right)_{i}$ of Filippov solutions $\xi_{t}(x)$ in $D$, each $\left(\xi_{t}\right)_{i}$ monotonically converges towards the projection of $\Phi(D)$ onto $\Omega_{i}$. This monotonic convergence property is summarized in the following lemma, see also [5], [3].

Lemma 2.1: For every regulatory domain $D \in \mathcal{D}_{r}$, all solutions $\xi_{t}$ in $D$ monotonically converge towards the focal set $\Phi(D)$. For every switching domain $D \in \mathcal{D}_{s}$, and every $i \in I$, the component $\left(\xi_{t}\right)_{i}$ of the solution $\xi_{t}$ in $D$ monotonically converges towards the closed interval

$$
\pi_{i}(\Phi(D))=\left\{\phi_{i} \in \Omega_{i} \mid \phi \in \Phi(D)\right\},
$$

the projection of $\Phi(D)$ onto $\Omega_{i}$, if $\left(\xi_{0}\right)_{i} \notin \pi_{i}(\Phi(D))$. This holds for all components $\left(\xi_{t}\right)_{i}$ of $\xi_{t}, 1 \leq i \leq n$.

The proof also gives the following corollary. By a slight abuse of notation, we will denote $\Pi(D)$ the smallest closed hyperrectangle in $\operatorname{supp}(D)$ containing $\Phi(D)$, that is the hyperrectangle the projection of which on the $i$-th axis is $\pi_{i}(\Phi(D))$ for all $i$.

Corollary 1: All solutions $\xi_{t}$ in $D$ converge towards $\Pi(D)$, if $\xi_{0} \notin \Pi(D)$. $\Pi(D)$ is positively invariant.

Corollary 2: If $\Phi(D)$ is a point, all solutions $\xi_{t}$ in $D$ converge monotonically towards $\Phi(D)$.

Moreover, we make a technical assumption on the focal sets for our system.

Assumption 1: For all domains $D \in \mathcal{D}$,

$$
\Phi(D) \cap \operatorname{supp}\left(D^{\prime}\right)=\{\}, \forall D^{\prime} \subseteq \partial D .
$$

For regulatory domains, Assumption 1 is equivalent to the assumption that $\phi(D) \notin \operatorname{supp}\left(D^{\prime}\right)$ for all $D^{\prime} \subseteq \partial D$.

Corollary 3: Under Assumption 1, for all domains $D \in$ $\mathcal{D}$,

$$
\Pi(D) \cap \operatorname{supp}\left(D^{\prime}\right)=\{\}, \forall D^{\prime} \subseteq \partial D .
$$

\section{EQUILIBRIA AND STABILITY}

\section{A. Regular Equilibrium Points}

It is clear that, if $D$ is a regulatory domain with focal point $\phi(D)$, and if $\phi(D) \in D$ then $x=\phi(D)$ is an asymptotically stable equilibrium point of (2).

Hence $(0,0)$ is a regular equilibrium point for the example network, and is asymptotically stable (see Figure 3). This stable regular equilibrium point represents a state of the network where both gene $a$ and gene $b$ are off.
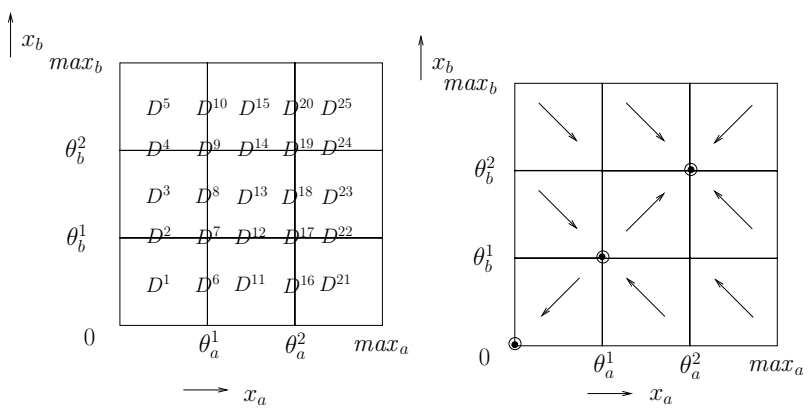

Fig. 3. (a) Phase space box $\Omega$ for the PL model in Figure 1 showing all the domains. (b) Sketch of the vector field for the PL model in Figure 1, with the parameter values from Section 2. There is a regular equilibrium point at $(0,0) \in D^{1}$ and there are singular equilibrium points in $D^{7}$ and $D^{19}$.

\section{B. Singular Equilibrium Points and Sets}

We say that a point $y \in \Omega$ is an equilibrium point for the differential inclusion if $0 \in H(y)$. In case that $y$ is contained in a switching domain, we call such a point a singular equilibrium point. Given a switching domain $D \in \mathcal{D}_{s}$, we can check for singular equilibrium points by computing the focal set $\Phi(D)$. Under Assumption 1, if $\Phi(D) \cap D \neq\{\}$ then we can deduce that $\Phi(D) \subseteq D$, i.e. that $D$ strictly contains or equals its focal set $(\Phi(D)$ being a closed set, equality 
only occurs if $D$ is a point). In fact, then every $\phi \in \Phi(D)$ is a singular equilibrium point of the differential inclusion. That is, for each $\phi \in \Phi(D)$, there exists a solution $\xi_{t}$ such that $\xi_{t}(\phi)=\phi, \forall t \geq 0$. Hence $\Phi(D)$ is a set of singular equilibrium points for the system, and we refer to such a $\Phi(D)$ as a singular equilibrium set.

In the example network of Figure 1, it can be seen from applying Definition 5 to the switching domain $D^{19}$ that the focal set $\Phi\left(D^{19}\right)$ is given by $D^{19}$ itself. In this example, $D^{19}$ has order 2 in a 2-dimensional phase space $\Omega$, so $D^{19}$ is simply a point and $\Phi\left(D^{19}\right)$ is a singleton. Hence $\Phi\left(D^{19}\right)$ is a singular equilibrium point and from examining the local behaviour of the vector field in Figure 3, it appears to be stable in some sense. Similarly, $\Phi\left(D^{7}\right)$ is a singular equilibrium point and from examining the local behaviour of the vector field, it appears to be a saddle-like point and hence unstable.

The fact that equilibria in switching domains can be setvalued, in addition to the non-uniqueness of solutions to differential inclusions, requires some extended definitions of stability. Filippov uses the term weakly stable to describe a equilibrium point that is stable in the sense of Lyapunov for some solution of the differential inclusion [9]. The term stable is reserved for a stronger concept of stability, and describes a equilibrium point that is stable in the sense of Lyapunov for every solution of the differential inclusion. Asymptotically stable and weakly asymptotically stable can be defined similarly. These concepts of stable and weakly stable equilibrium points can be extended naturally to define stability for equilibrium sets (cf. [3] for precise definitions).

\section{GRaph of the Qualitative Dynamics}

De Jong et al have developed a discrete, qualitative description of the dynamics of the PL system that underlies the qualitative simulation of genetic regulatory networks [5]. The state transition graph is a discrete representation consisting of the qualitative states of the system (the domains) and all possible transitions between them. This description is key to formulating and proving the results on stability of singular equilibrium points in Section V.

\section{A. States and Transitions}

The set of domains $\mathcal{D}$ can be thought of as qualitative states, since the PL system (2) behaves in a qualitatively homogeneous manner in each domain $D \in \mathcal{D}$.

Given two contiguous domains $D, D^{\prime} \in \mathcal{D}$, we say that there is transition from $D$ to $D^{\prime}$ if there is a solution in the sense of Filippov lying in $D$ and terminating in $D^{\prime}$. We define a transition from $D$ to $D^{\prime}$ more precisely below. Recall that $A(D) \subset \mathcal{D}_{s}$ is the set of all domains in the boundary of $D$.

Definition 6: Let $D, D^{\prime} \in \mathcal{D}$ be two contiguous domains. We say that there exists a transition from $D$ to $D^{\prime}$ if one of the following two properties holds:

1) If $D^{\prime} \in A(D)$, then there exists $x_{0} \in D$ and a Filippov solution $\xi_{t}\left(x_{0}\right)$ defined on a finite time interval $[0, \tau]$ such that

a) $\xi_{t}\left(x_{0}\right) \in D$ for all $t \in[0, \tau)$, and b) $\xi_{\tau}\left(x_{0}\right) \in D^{\prime}$.

2) If $D \in A\left(D^{\prime}\right)$, then there exists $x_{0} \in D$ and a Filippov solution $\xi_{t}\left(x_{0}\right)$ defined on a finite time interval $[0, \tau]$ such that

a) $\xi_{0}\left(x_{0}\right)=x_{0} \in D$, and

b) $\xi_{t}\left(x_{0}\right) \in D^{\prime}$ for all $t \in(0, \tau]$.

We can prove the following two properties of transitions.

Proposition 4.1: Let $D, D^{\prime} \in \mathcal{D}$ be two contiguous domains such that $D^{\prime} \in A(D)$. Under Assumption 1, there exists a transition from $D$ to $D^{\prime}$ iff (1) $\Phi(D) \neq\{\}$ and (2) for all $i \in\{1, \ldots, n\}$ such that $x_{i}$ is switching in $D^{\prime}$ but not in $D$,

$$
\left(d^{\prime}{ }_{i}-d_{i}\right)\left(\phi_{i}-d^{\prime}{ }_{i}\right)>0, \forall d \in D, \forall d^{\prime} \in D^{\prime}, \forall \phi \in \Phi(D) .
$$

The proof is not detailed here: it is based on the fact that Assumption 1 implies that, if there is a transition, $\Phi(D)$ is "on the other side" of $D$ with respect to $D^{\prime}$.

Proposition 4.2: Let $D, D^{\prime} \in \mathcal{D}$ be two contiguous domains such that $D \in A\left(D^{\prime}\right)$. Under Assumption 1, there exists a transition from $D$ to $D^{\prime}$ iff (1) $\Phi\left(D^{\prime}\right) \neq\{\}$ and (2) for all $i \in\{1, \ldots, n\}$ such that $x_{i}$ is switching in $D$ but not in $D^{\prime}$,

$$
\left(d^{\prime}{ }_{i}-d_{i}\right)\left(\phi_{i}^{\prime}-d_{i}\right)>0, \forall d \in D, \forall d^{\prime} \in D^{\prime}, \forall \phi^{\prime} \in \Phi\left(D^{\prime}\right) .
$$

Proof: Similar to the proof of Proposition 4.1.

\section{B. State Transition Graph}

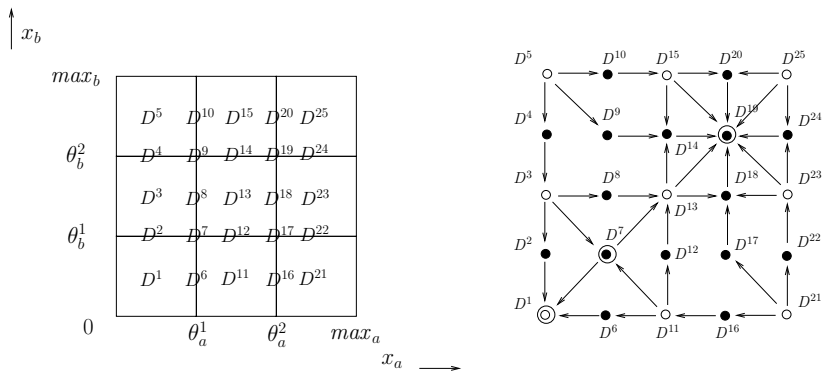

Fig. 4. Phase space and state transition graph for the PL model in Figure 1. Nodes associated with regulatory and switching domains are indicated by unfilled and filled dots, respectively. Domains containing equilibria are additionally circled.

A state transition graph (STG) is a directed graph whose vertices are the domains of the system and whose edges are the transitions between these domains. The state transition graph is a discrete representation of the qualitative dynamics of the piecewise-linear system. Figure 4 shows the state transition graph for the example two-gene network from Figure 1. The domains represented by the nodes in the state transition graph can be thought of as qualitative states of the PL model.

Recall that within each domain, i.e. for each vertex of the STG, the PL system (2) behaves in a qualitatively homogeneous way. Hence many dynamical properties of the full PL system can be analyzed simply by studying the STG. Since the STG captures the essential qualitative dynamics of the PL 
system, it is useful to prove results on stability of equilibria that can be inferred directly from the STG. For example, for the two-gene network, the sequence $\left\langle D^{21}, D^{17}, D^{18}, D^{19}\right\rangle$ is a path in the STG shown in Figure 4. This path corresponds to solutions with initial conditions in domain $D^{21}$ that reach a qualitative equilibrium state in $D^{19}$, corresponding to a domain containing an equilibrium point. Whether such qualitative equilibrium states represent equilibria that are stable is investigated in the next section.

\section{CRiteria For Stability of Singular Equilibria}

As discussed in Section III, regular equilibrium points are known to be asymptotically stable so we focus instead on the stability of equilibria located in switching domains: singular equilibrium points and sets. We work under the caveat that Assumption 1 from Section II-C holds for every domain D in our system.

The proof in $\mathbb{R}^{2}$ is included since it gives a geometrical intuition for the general proof in $\mathbb{R}^{n}$.

\section{A. Theorem on Stability}

The following theorems allow us to link the rigorous concepts of Lyapunov stability for Filippov solutions (cf. [3] for precise definitions) with the qualitative dynamics of the system represented by the state transition graph. Theorems 5.1 and 5.2 state that a switching domain $D$ contains an asymptotically stable equilibrium set if every contiguous regulatory domain has a transition entering the domain $D$. The first theorem is stated and proved for $\Omega \subset \mathbb{R}^{2}$, and the second is the general case for $p$ switching variables in $\Omega \subset \mathbb{R}^{n}$.

Theorem 5.1: Assume $\Omega \subset \mathbb{R}^{2}$. Let $D \in \mathcal{D}_{s}$ be a switching domain containing a singular equilibrium point $\Phi(D)$. If for all $D^{\prime} \in R(D)$ there exists a transition from $D^{\prime}$ to $D$ in the state transition graph $G$, then $\Phi(D)$ is asymptotically stable.

Proof: Let $x=\left(x_{1}, x_{2}\right) \in D$. In $\mathbb{R}^{2}$ there are only two cases: one of the variables is switching, or both are switching.

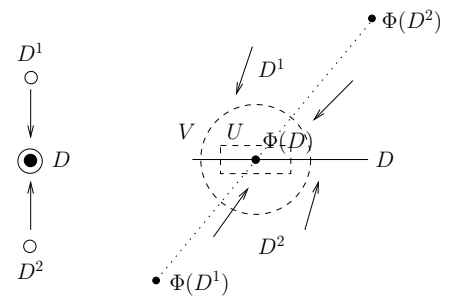

Fig. 5. State transition graph and sketch of phase portrait for case 1 in the proof of Theorem 5.1.

Case 1: Assume $x_{2}$ is a switching variable on $D$ and $x_{1}$ is not. The subgraph of $G$ showing $D$ and $R(D)$ can be seen in Figure 5, and the phase portrait showing the neighbourhoods. The detailed proof is in [3]).

Case 2: Assume both $x_{1}$ and $x_{2}$ are switching variables on $D$. Then there are four regulatory domains in $R(D)$ and we have the situation shown in Figure 6. For each regulatory domain $D^{\prime} \in R(D)=\left\{D^{1}, D^{3}, D^{7}, D^{9}\right\}$, we can extend
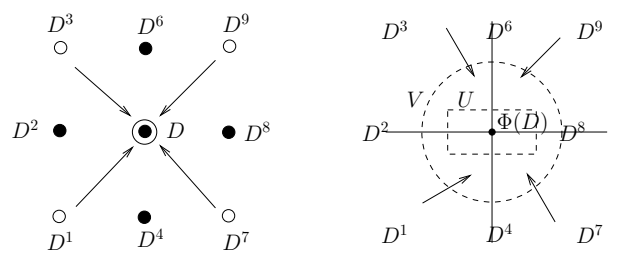

Fig. 6. State transition graph and sketch of phase portrait for case 2 in the proof of Theorem 5.1. Only transitions from $D^{\prime} \in R(D)$ are shown.

the arguments used in case 1 above to deduce that $\phi\left(D^{i}\right)$ $(i=1,3,7,9)$ lie in a specific quadrant. For example, the transition from $D^{3}$ to $D$ and Proposition 4.1 implies that $\phi\left(D^{3}\right)$ lies in the quarter-space defined by $\left\{x \in \Omega \mid x_{1}>\right.$ $\left.\theta_{1}^{q_{1}}, x_{2}<\theta_{2}^{q_{2}}\right\}$. This can be seen as follows. Let $D=\{x \in$ $\left.\Omega \mid x_{1}=\theta_{1}^{q_{1}}, x_{2}=\theta_{2}^{q_{2}}\right\}, D^{3}=\left\{x \in \Omega \mid \theta_{1}^{q_{1}-1}<x_{1}<\right.$ $\left.\theta_{1}^{q_{1}}, \theta_{2}^{q_{2}}<x_{2}<\theta_{2}^{q_{2}+1}\right\}$, and $D^{7}=\left\{x \in \Omega \mid \theta_{1}^{q_{1}}<x_{1}<\right.$ $\left.\theta_{1}^{q_{1}+1}, \theta_{2}^{q_{2}-1}<x_{2}<\theta_{2}^{q_{2}}\right\}$. Then $D^{3}$ and $D^{7}$ are regulatory domains as illustrated in Figure 6. By hypothesis, there is a transition from $D^{3}$ to $D$ in the STG, so it follows from Proposition 4.1 that

$$
\begin{aligned}
& \left(d_{1}-d_{1}^{3}\right)\left(\phi_{1}^{3}-d_{1}\right)>0, \forall d \in D, \forall d^{3} \in D^{3}, \\
& \left(d_{2}-d_{2}^{3}\right)\left(\phi_{2}^{3}-d_{2}\right)>0, \forall d \in D, \forall d^{3} \in D^{3},
\end{aligned}
$$

where $\phi^{3}=\phi\left(D^{3}\right)$. Since $d_{2}<d_{2}^{3}, \forall d \in D, \forall d^{3} \in D^{3}$, (15) implies that the focal point $\phi\left(D^{3}\right)$ lies somewhere in the half-space defined by $\left\{x \in \Omega \mid x_{2}<\theta_{2}^{q_{2}}\right\}$. Similarly, since $d_{1}^{3}<d_{1}, \forall d \in D, \forall d^{3} \in D^{3}$, (14) implies that the focal point $\phi\left(D^{3}\right)$ lies somewhere in the half-space defined by $\left\{x \in \Omega \mid x_{1}>\theta_{1}^{q_{1}}\right\}$. Thus, (14) and (15) together imply that

$$
\phi\left(D^{3}\right) \in\left\{x \in \Omega \mid x_{1}>\theta_{1}^{q_{1}}\right\} \cap\left\{x \in \Omega \mid x_{2}<\theta_{2}^{q_{2}}\right\},
$$

and every solution, $\xi_{t}=\left(x_{1}(t), x_{2}(t)\right)$, starting in $D^{3}$ has the property $\dot{x}_{1}>0$ and $\dot{x}_{2}<0$. Taking a sector $U^{3}$ in $D^{3}$ that will form part of a neighbourhood of $\Phi(D)$, all solutions starting in $U^{3}$ will either reach one of the switching domains $D^{2}$ or $D^{6}$ or go directly to $D$. The transition from $D^{3}$ and $D^{1}$ to $D$ in the STG result in sliding modes along $D^{2}$, with focal set $\Phi\left(D^{2}\right)$ such that $\Phi\left(D^{2}\right) \subseteq$ $\left\{x \in \Omega \mid x_{1}>\theta_{1}^{q_{1}}\right\}$. This is because $\Phi\left(D^{2}\right)$ is defined using a convex combination of $\phi\left(D^{1}\right)$ and $\phi\left(D^{3}\right)$, both of which lie in the region $\Phi\left(D^{2}\right) \subseteq\left\{x \in \Omega \mid x_{1}>\theta_{1}^{q_{1}}\right\}$, hence $\Phi\left(D^{2}\right)$ will also lie in this region. It follows that solutions starting in $U^{3}$ that reach $D^{2}$ will slide along $D^{2}$ until they reach $D$. Similarly, solutions starting in $U^{3}$ that reach $D^{6}$ will slide along $D^{6}$ until they reach $D$. Analogous regions can be constructed in the other regulatory domains, $\left\{D^{1}, D^{7}, D^{9}\right\}$. From these sectors, $\left\{U^{1}, U^{3}, U^{7}, U^{9}\right\}$, in the domains $\left\{D^{1}, D^{3}, D^{7}, D^{9}\right\}$, we can construct a neighbourhood $U$ of $\Phi(D)$. Given any neighbourhood $V$ of $\Phi(D)$, we can construct such a neighbourhood $U \subset V$. From Lemma 2.1 , the monotonic convergence of solutions means that all solutions starting in a small enough $U$ will remain in $V$. The above reasoning shows that all these solutions eventually converge to $D$ and hence to $\Phi(D)$. This completes the proof of Theorem 5.1. 
Below we state the general result in $\mathbb{R}^{n}$ for $p$ switching variables. The details are similar to the two dimensional case (see [3]).

Theorem 5.2: Assume $\Omega \subset \mathbb{R}^{n}$. Let $D \in \mathcal{D}_{s}$ be a switching domain of order $p>1$ containing a singular equilibrium set $\Phi(D)$ that satisfies Assumption 1. If for all $D^{\prime} \in R(D)$, there is a transition from $D^{\prime}$ to $D$ in the state transition graph, then $\Phi(D)$ is weakly asymptotically stable and $\Pi(D)$ is asymptotically stable.

Corollary 4: Under the conditions of Theorem 5.2, if, moreover, $\Phi(D)$ is a point, it is asymptotically stable.

There is no hope for proving more in the case when $\Phi(D)$ is a set. To see that, let us suppose that $n=4$, underwhich condition $\Phi(D)$ can be a segment when $D$ is of dimension 2. If this segment is not parallel to the axes, and if the $\gamma_{i}$ are not all equal, then there exist solutions starting from one point $\phi_{1}$ of the segment and leaving the segment (but staying in the rectangle $\Pi(D)$ ) to converge towards another point $\phi_{2}$ of the segment.

In the two-gene network of Figure 1, the switching domain $D^{19}$ satisfies the criteria of Theorems 5.1 or 5.2 , since each domain $D^{\prime} \in R(D)=\left\{D^{13}, D^{15}, D^{23}, D^{25}\right\}$ has a transition from $D^{\prime}$ to $D^{19}$. Hence Theorems 5.1 or 5.2 show that $D^{19}$ contains an asymptotically stable singular equilibrium point.

\section{Discussion}

Assumption 1 is crucial to most of the results presented here. Assumption 1 is generic if the focal set is a point (i.e. for regulatory domains or for switching domains in $\mathbb{R}^{2}$ ), in the sense that a specific relationship between the independent parameters $\left\{\theta_{i}^{j}\right\},\left\{\gamma_{i}\right\},\left\{\kappa_{i l}\right\}$ has to be specified for Assumption 1 to be violated. However, in the case when $\Phi(D)$ is a set, Assumption 1 is not generic anymore.

The qualitative simulation of genetic regulatory networks, leading to the generation of the state transition graph for PL models, has been implemented in the software tool Genetic Network Analyzer (GNA). GNA has been used in collaboration with experimental biologists for the analysis of several genetic regulatory networks of biological interest: the initiation of sporulation in Bacillus subtilis [4], quorum sensing in Pseudomonas aeruginosa [21], and the nutritional stress response in Escherichia coli [18]. Based on the work presented in this paper, a module of GNA for identification of all regular and singular equilibria and determination of their stability could be developed.

Acknowledgments. The authors acknowledge the financial support of the ARC GDyn initiative at INRIA, the ACI IMPBio BacAttract initiative at the French Ministry for Research, and the European project NEST HYGEIA.

\section{REFERENCES}

[1] R. Alur, C. Belta, F. Ivančíc, V. Kumar, M. Mintz, G. Pappas, H. Rubin, and J. Schlug. Hybrid modeling and simulation of biomolecular networks. In M. D. Benedetto and A. Sangiovanni-Vincentelli, editors, Hybrid Systems: Computation and Control (HSCC 2001), volume 2034 of LNCS, pages 19-32. Springer-Verlag, 2001.
[2] C. Belta, P. Finin, L. C. G. J. M. Habets, A. Halasz, M. Imielinksi, V. Kumar, and H. Rubin. Understanding the bacterial stringent response using reachability analysis of hybrid systems. In R. Alur and G. Pappas, editors, Hybrid Systems: Computation and Control (HSCC 2004), volume 2993 of LNCS. Springer-Verlag, Berlin, 2004.

[3] R. Casey, H. de Jong, and J.-L. Gouzé. Piecewise-linear models of genetic regulatory networks: Equilibria and their stability. Technical Report RR-5353, INRIA, 2004, to appear in J. Math. Biol.

[4] H. de Jong, J. Geiselmann, G. Batt, C. Hernandez, and M. Page. Qualitative simulation of the initiation of sporulation in Bacillus subtilis. Bull. Math. Biol., 6(2):261-299, 2004.

[5] H. de Jong, J.-L. Gouzé, C. Hernandez, M. Page, T. Sari, and J. Geiselmann. Qualitative simulation of genetic regulatory networks using piecewise-linear models. Bull. Math. Biol., 6(2):301-340, 2004.

[6] R. Decarlo, M. Branicky, S. Pettersson, and B. Lennartson. Perspectives and results on the stability and stabilizability of hybrid systems. Proc. IEEE, 88(7):1069-1083, 2000.

[7] C. Edwards and S. Spurgeon. Sliding Mode Control: Theory and Applications. Taylor \& Francis, 1998.

[8] R. Edwards. Analysis of continuous-time switching networks. Physica D, 146:165-199, 2000.

[9] A. F. Filippov. Differential Equations with Discontinuous Righthand Sides. Kluwer Academic Publishers, Dordrecht, 1988.

[10] R. Ghosh and C. Tomlin. Lateral inhibition through Delta-Notch signaling: A piecewise affine hybrid model. In M. D. Benedetto and A. Sangiovanni-Vincentelli, editors, Hybrid Systems: Computation and Control (HSCC 2001), volume 2034 of LNCS, pages 232-246. Springer-Verlag, Berlin, 2001.

[11] L. Glass and S. A. Kauffman. The logical analysis of continuous non-linear biochemical control networks. J. Theor. Biol., 39:103-129, 1973.

[12] L. Glass and J. Pasternack. Stable oscillations in mathematical models of biological control systems. J. Math. Biol., 6:207-223, 1978.

[13] J. Gouzé and T. Sari. A class of piecewise linear differential equations arising in biological models. Dyn. Syst, 17(4):299-316, 2002.

[14] K. Kohn. Molecular interaction maps as information organizers and simulation guides. Chaos, 11(1):1-14, 2001.

[15] T. Mestl, E. Plahte, and S. Omholt. A mathematical framework for describing and analysing gene regulatory networks. J. Theor. Biol., 176(2):291-300, 1995.

[16] E. Plahte, T. Mestl, and S. Omholt. Global analysis of steady points for systems of differential equations with sigmoid interactions. Dyn. Stabil. Syst., 9(4):275-291, 1994.

[17] M. Ptashne. A Genetic Switch: Phage $\lambda$ and Higher Organisms. Cell Press \& Blackwell Science, Cambridge, MA, 2nd edition, 1992.

[18] D. Ropers, H. de Jong, M. Page, D. Schneider, and H. Geiselmann. Qualitative simulation of the nutritional stress response in Escherichia coli. Technical Report RR-5412, INRIA, 2004, to appear in Biosystems.

[19] R. Thomas, D. Thieffry, and M. Kaufman. Dynamical behaviour of biological regulatory networks: I. Biological role of feedback loops and practical use of the concept of the loop-characteristic state. Bull. Math. Biol., 57(2):247-276, 1995.

[20] V. I. Utkin. Sliding Modes in Control and Optimization. Communications and Control Engineering. Springer-Verlag, Berlin, 1992.

[21] A. U. Viretta and M. Fussenegger. Modeling the quorum sensing regulatory network of human-pathogenic Pseudomonas aeruginosa. Biotech. Prog., 20:670-678, 2004. 